\title{
THE PÓLYA-AEPPLI PROCESS AND RUIN PROBLEMS
}

\author{
LEDA D. MINKOVA
}

Received 16 September 2003 and in revised form 21 May 2004

The Pólya-Aeppli process as a generalization of the homogeneous Poisson process is defined. We consider the risk model in which the counting process is the Pólya-Aeppli process. It is called a Pólya-Aeppli risk model. The problem of finding the ruin probability and the Cramér-Lundberg approximation is studied. The Cramér condition and the Lundberg exponent are defined. Finally, the comparison between the Pólya-Aeppli risk model and the corresponding classical risk model is given.

\section{Introduction}

The standard model of an insurance company, called risk process $\{X(t), t \geq 0\}$, defined on the complete probability space $(\Omega, \mathscr{F}, P)$, is given by

$$
X(t)=c t-\sum_{k=1}^{N(t)} Z_{k}, \quad\left(\sum_{1}^{0}=0\right) .
$$

Here $c$ is a positive real constant representing the gross risk premium rate. The sequence $\left\{Z_{k}\right\}_{k=1}^{\infty}$ of mutually independent and identically distributed random variables (r.v.'s) with common distribution function $F, F(0)=0$, and mean value $\mu$ is independent of the counting process $N(t), t \geq 0$. The process $N(t)$ is interpreted as the number of claims on the company during the interval $[0, t]$.

In the classical risk model, the process $N(t)$ is a stationary Poisson counting process; see for instance Grandell [4]. In this case, the aggregate claim amounting up to time $t$ is given by the compound Poisson process $S(t)=\sum_{i=1}^{N(t)} Z_{i}$. If the number of claims $N(t)$ forms a renewal counting process, the model (1.1) is called a renewal risk model. There are many directions in which the classical risk model and the renewal model are generalized in order to become a reasonably realistic description. Dickson [2] studied a generalization of the renewal model, assuming that claims occur as an Erlang process, and extended several classical results. References are given in Asmussen [1] and Rolski et al. [9]. Our interest is in the generalization of the counting process $N(t)$. In $[7,8]$, a new

Copyright (C) 2004 Hindawi Publishing Corporation Journal of Applied Mathematics and Stochastic Analysis 2004:3 (2004) 221-234 2000 Mathematics Subject Classification: 60K10, 62P05

URL: http://dx.doi.org/10.1155/S1048953304309032 
family of discrete probability distributions is introduced. The classical Poisson, negative binomial, binomial, and logarithmic distributions are generalized by adding a new parameter $\rho \in[0,1)$. The generalized distributions are called inflated-parameter distributions according to the interpretation of the parameter $\rho$. The new family of distributions is called inflated-parameter generalized power series distributions (IGPSD). In the case of $\rho=0$, the IGPSD becomes the family of generalized power series distributions (GPSD) or the classical discrete distributions. A natural question is: what will be the corresponding generalization of the classical risk model?

We give useful interpretation of the model. Suppose that any insurance policy produces two types of claims named "success" with probability $1-\rho$ and "failure" with probability $\rho$. Define the r.v. $N$ to equal the number of trials until the $i$ th successive claim appears. The r.v. $N$ is negative binomial distributed with parameters $1-\rho$ and $i$. The probability mass function of $N$ is given by

$$
P(N=k)=\left(\begin{array}{c}
k-1 \\
k-i
\end{array}\right) \rho^{k-i}(1-\rho)^{i}, \quad k=i, i+1, \ldots
$$

The r.v. $N$, given by (1.2), can be represented as a sum $N=X_{1}+\cdots+X_{i}$, where $\left\{X_{j}\right.$, $j=1,2, \ldots\}$ are independent identically $G e_{1}(1-\rho)$-distributed r.v.'s. The parameter $i$ in (1.2) represents the number of geometrically distributed r.v.s. If we suppose that $i$ is an outcome of the r.v. $Y$, independent of $\left\{X_{j}, j=1,2, \ldots\right\}$, and $Y$ has the GPSD, then $N$ has the IGPSD; see [8]. In particular, if $Y$ has the Poisson distribution with parameter $\lambda(\operatorname{Po}(\lambda)), N$ has the inflated-parameter Poisson distribution $(\operatorname{IPo}(\lambda, \rho))$. The $\operatorname{IPo}(\lambda, \rho)$ distribution coincides with the Pólya-Aeppli distribution (see [5]) and has the following probability mass function:

$$
P(N=n)= \begin{cases}e^{-\lambda}, & n=0, \\
e^{-\lambda} \sum_{i=1}^{n}\left(\begin{array}{c}
n-1 \\
i-1
\end{array}\right) \frac{[\lambda(1-\rho)]^{i}}{i !} \rho^{n-i}, & n=1,2, \ldots\end{cases}
$$

In the next section, we will define the Pólya-Aeppli process in order to describe the aggregate claim amount process as a compound Pólya-Aeppli process. Section 3 deals with the risk model in the case of Pólya-Aeppli counting process. The ruin probability in two cases, ordinary and stationary, is studied. In Section 4, the Cramér-Lundberg approximation is given. A comparison between the Pólya-Aeppli risk model and the corresponding classical risk model is given in Section 5.

\section{The Pólya-Aeppli process}

The $\operatorname{IPo}(\lambda, \rho)$ distribution is a generalization of the classical $\operatorname{Po}(\lambda)$ distribution. In this section, we will define the corresponding generalization of the Poisson process.

We consider the sequence $T_{1}, T_{2}, \ldots$ of nonnegative, mutually independent r.v.'s and the corresponding renewal process

$$
S_{n}=\sum_{k=1}^{n} T_{k}, \quad n=1,2, \ldots, S_{0}=0 .
$$


The process $S_{n}$ can be interpreted as a sequence of renewal epochs. $T_{1}$ is the time until the first renewal epoch and $\left\{T_{k}\right\}_{k \geq 2}$ are the interarrival times.

Let $N(t)=\sup \left\{n \geq 0, S_{n} \leq t\right\}, t \geq 0$, be the number of renewals occurring up to time $t$. The distribution of $N(t)$ is related to that of $S_{n}$, and for any $t \geq 0$ and $n \geq 0$, the following probability relation holds:

$$
P(N(t)=n)=P\left(S_{n} \leq t\right)-P\left(S_{n+1} \leq t\right), \quad n=0,1,2, \ldots
$$

We will suppose that $N(t)$ is described by the $\operatorname{IPo}(\lambda t, \rho)$ distribution (or Pólya-Aeppli distribution), with mean function $(\lambda /(1-\rho)) t$, that is,

$$
P(N(t)=n)= \begin{cases}e^{-\lambda t}, & n=0, \\
e^{-\lambda t} \sum_{i=1}^{n}\left(\begin{array}{c}
n-1 \\
i-1
\end{array}\right) \frac{[\lambda(1-\rho) t]^{i}}{i !} \rho^{n-i}, & n=1,2, \ldots\end{cases}
$$

We denote by $\operatorname{LS}_{X}(s)=\int_{0}^{\infty} e^{-s x} d F_{X}(x)$ the Laplace-Stieltjes transform (LST) of any r.v. $X$ with distribution function $F_{X}(x)$. Let $p_{n}(t)=P(N(t)=n)$.

For the next considerations, we need the following result.

LEMMA 2.1. The LST of $p_{n}(t)$ is given by

$$
\mathrm{LS}_{p_{n}(t)}(s)=\int_{0}^{\infty} e^{-s t} d p_{n}(t)= \begin{cases}-\frac{\lambda}{s+\lambda}, & n=0, \\ (1-\rho) \frac{\lambda}{s+\lambda} \frac{s}{s+\lambda}\left[\rho+(1-\rho) \frac{\lambda}{s+\lambda}\right]^{n-1}, & n=1,2, \ldots\end{cases}
$$

Now we will show that the renewal process is characterized by the fact that $T_{1}$ is exponentially distributed and $\left\{T_{2}, T_{3}, \ldots\right\}$ are identically distributed. Moreover, $T_{2}$ is zero with probability $\rho$, and with probability $1-\rho$, exponentially distributed with parameter $\lambda$. This means that the probability density functions and the mean values are the following:

$$
\begin{gathered}
f_{T_{1}}(t)=\lambda e^{-\lambda t}, \quad t \geq 0, \quad E T_{1}=\frac{1}{\lambda} \\
f_{T_{2}}(t)=\rho \delta_{0}(t)+(1-\rho) \lambda e^{-\lambda t}, \quad t \geq 0, \quad E T_{2}=\frac{1-\rho}{\lambda},
\end{gathered}
$$

where

$$
\delta_{0}(t)= \begin{cases}1, & \text { if } t=0 \\ 0, & \text { otherwise }\end{cases}
$$

The process $S_{n}$ is called a delayed renewal process with a delay $T_{1}$.

THEOREM 2.2. There exists exactly one renewal process such that the number of renewals up to time thas the Pólya-Aeppli distribution (2.3). In this case, the time until the first renewal 
224 The Pólya-Aeppli process and ruin problems

epoch $T_{1}$ is exponentially distributed with parameter $\lambda$. The interarrival times $T_{2}, T_{3}, \ldots$ are zero with probability $\rho$, and with probability $1-\rho$, exponentially distributed with parameter $\lambda$.

Proof. To prove the theorem, it suffices to show that the LST of the r.v. $S_{n}$ is as follows:

$$
\mathrm{LS}_{S_{n}}(s)=\frac{\lambda}{s+\lambda}\left[\rho+(1-\rho) \frac{\lambda}{s+\lambda}\right]^{n-1}
$$

We will prove it by induction using the relations (2.2). For $n=0,(2.2)$ becomes

$$
P(N(t)=0)=1-P\left(T_{1} \leq t\right)=1-F_{T_{1}}(t),
$$

where $F_{T_{1}}(t)$ is the distribution function of $T_{1}$. On the other hand, from (2.3), it follows that

$$
P(N(t)=0)=e^{-\lambda t}
$$

Combining (2.9) and (2.10) gives that $F_{T_{1}}(t)=1-e^{-\lambda t}$, that is, the r.v. $T_{1}$ is exponentially distributed with parameter $\lambda$ and $\operatorname{LST} \lambda /(s+\lambda)$.

Now from (2.2), for $n=1$, we get

$$
P(N(t)=1)=P\left(S_{1} \leq t\right)-P\left(S_{2} \leq t\right) .
$$

Taking the LST leads to

$$
(1-\rho) \frac{\lambda}{s+\lambda} \frac{s}{s+\lambda}=\mathrm{LS}_{S_{1}}(s)-\mathrm{LS}_{S_{2}}(s)
$$

After some algebra, we arrive at

$$
\operatorname{LS}_{T_{1}+T_{2}}(s)=\frac{\lambda}{s+\lambda}\left[\rho+(1-\rho) \frac{\lambda}{s+\lambda}\right]
$$

which means that $T_{2}$ is independent of $T_{1}$. Moreover, $T_{2}$ is an exponentially distributed r.v. with parameter $\lambda$ and mass at zero equal to $\rho$. The probability density function of $T_{2}$ is given by (2.6).

Suppose now that for any $n \geq 2$, the LST of $S_{n}$ is given by (2.8). Taking the LST in (2.2), we get

$$
\begin{aligned}
\operatorname{LS}_{S_{n+1}}(s) & =\int_{0}^{\infty} e^{-s t} d P\left(S_{n+1} \leq t\right) \\
& =\int_{0}^{\infty} e^{-s t} d P\left(S_{n} \leq t\right)-\int_{0}^{\infty} e^{-s t} d p_{n}(t)
\end{aligned}
$$


Applying Lemma 2.1, one can show that the LST of the renewal process $S_{n+1}$ is equal to

$$
\operatorname{LS}_{S_{n+1}}(s)=\frac{\lambda}{s+\lambda}\left[\rho+(1-\rho) \frac{\lambda}{s+\lambda}\right]^{n}
$$

This proves the theorem.

Remark 2.3. In the case of $\rho=0$, the LST (2.8) becomes the LST of Gamma $(n, \lambda)$ (or Erlang $(n))$ distributed r.v. This case coincides with the usual homogeneous Poisson process.

Remark 2.4. We note that the probability distribution function of $T_{2}$ is given by

$$
F_{T_{2}}(t)=1-(1-\rho) e^{-\lambda t}, \quad t \geq 0 .
$$

That family of distributions has a jump at zero, that is, $P\left(T_{2}=0\right)=\rho$.

Remark 2.5. It is easy to see that the exponential distribution function of the delay, $F_{T_{1}}(t)$, and the distribution function $F_{T_{2}}(t)$ satisfy the following relation:

$$
F_{T_{1}}(t)=\frac{1}{E T_{2}} \int_{0}^{t}\left[1-F_{T_{2}}(u)\right] d u \text {. }
$$

In this case, the delayed renewal counting process is the only stationary renewal process; see [6]. From the renewal theory, it is known that under condition (2.17), the delayed renewal counting process has stationary increments; see for instance Rolski et al. [9, Theorem 6.1.8].

We proved the theorem using the LST and basic relation (2.2). The converse theorem is also true.

Theorem 2.6. Suppose that the interarrival times $\left\{T_{k}\right\}_{k \geq 2}$ of the stationary renewal process are equal to zero with probability $\rho$, and with probability $1-\rho$, exponentially distributed with parameter $\lambda$. Then the number of renewals up to time thas the Pólya-Aeppli distribution.

Now we can define the inflated-parameter Poisson process, or the Pólya-Aeppli process.

Definition 2.7. A counting process $\{N(t), t \geq 0\}$ is said to be a Pólya-Aeppli process if

(a) it starts at zero, $N(0)=0$;

(b) it has independent, stationary increments;

(c) for each $t>0, N(t)$ is Pólya-Aeppli distributed.

The Pólya-Aeppli process is a time-homogeneous process. In the case of $\rho=0$, it becomes a homogeneous Poisson process. So, we have a homogeneous process with an additional parameter. The additional parameter $\rho$ has an interpretation as a correlation coefficient; see [8]. 


\section{The Pólya-Aeppli risk model}

We consider the risk process $X$, defined by (1.1), where $N(t)$ is the Pólya-Aeppli process independent of the claim sizes $\left\{Z_{k}\right\}_{k=1}^{\infty}$. We will call this process a Pólya-Aeppli risk process.

The relative safety loading $\theta$ is defined by

$$
\theta=\frac{c(1-\rho)-\lambda \mu}{\lambda \mu}=\frac{c(1-\rho)}{\lambda \mu}-1,
$$

and in the case of positive safety loading $\theta>0, c>\lambda \mu /(1-\rho)$.

The survival probability, or nonruin probability $\Phi(u)$, of a company having initial capital $u$ is given by

$$
\Phi(u)=P\left\{\inf _{t \geq 0} X(t) \geq-u\right\}
$$

The ruin probability is defined by the equality $\Psi(u)=1-\Phi(u)$. We suppose that $u \geq 0$.

The occurrence of the claims in the risk process (1.1) is described by a delayed renewal counting process. We will study the ruin probability in two cases following the renewal arguments described by Feller [3] and Grandell [4].

3.1. The ordinary case. We suppose that the first claim has occurred and the subsequent claims occur as an ordinary renewal process. The interoccurrence times $T_{k}, k=1,2, \ldots$, are exponentially distributed with mass at zero equal to $\rho$ and probability density function given by (2.6). The claim sizes $Z_{1}, Z_{2}, \ldots$ are independent and identically distributed r.v.'s with common distribution function $F(x)$ with $F(0)=0$ and mean value $\mu$. Let

$$
F_{I}(x)=\frac{1}{\mu} \int_{0}^{x}[1-F(z)] d z
$$

be the integrated tail distribution. We define the function

$$
H(z)=\rho F(z)+\frac{\lambda \mu}{c} F_{I}(z)
$$

and note that

$$
H(\infty)=\rho F(\infty)+\frac{\lambda}{c} \int_{0}^{\infty}[1-F(z)] d z=\rho+\frac{\lambda \mu}{c}<1
$$

If we denote by $\Phi^{0}(u)$ and $\Psi^{0}(u)$ the nonruin and ruin probabilities, respectively, in the ordinary case, then the following result holds.

Proposition 3.1. The nonruin function $\Phi^{0}(u)$ satisfies the integral equation

$$
\Phi^{0}(u)=\Phi^{0}(0)+\int_{0}^{u} \Phi^{0}(u-z) d H(z), \quad u \geq 0
$$

where $H(z)$ is defined by (3.4). 
Proof. Suppose that the first claim occurs at epoch $s$. For no ruin to occur according to the renewal argument, we get

$$
\begin{aligned}
\Phi^{0}(t) & =\int_{0-}^{\infty}\left[\rho \delta_{0}+(1-\rho) \lambda e^{-\lambda s}\right] \int_{0}^{t+c s} \Phi^{0}(t+c s-z) d F(z) d s \\
& =\rho \int_{0-}^{t} \Phi^{0}(t-z) d F(z)+(1-\rho) \int_{0-}^{\infty} \lambda e^{-\lambda s} \int_{0}^{t+c s} \Phi^{0}(t+c s-z) d F(z) d s, \quad t \geq 0 .
\end{aligned}
$$

Change of variables and differentiation leads to

$$
\Phi^{0^{\prime}}(t)=\rho \Phi^{0}(0) F^{\prime}(t)+\rho \int_{0-}^{t} \Phi^{0^{\prime}}(t-z) d F(z)+\frac{\lambda}{c}\left[\Phi^{0}(t)-\frac{\lambda}{c} \int_{0}^{t} \Phi^{0}(t-z) d F(z)\right]
$$

where $\Phi^{0^{\prime}}(t)$ is the derivative of $\Phi^{0}(t)$. Integrating (3.8) in $t$ over $[0, u]$ and performing integration by parts, one gets

$$
\Phi^{0}(u)=\Phi^{0}(0)+\rho \int_{0-}^{u} \Phi^{0}(u-z) d F(z)+\frac{\lambda}{c} \int_{0}^{u} \Phi^{0}(u-z)[1-F(z)] d z,
$$

which is just (3.6).

COROLLARY 3.2. The ruin probability $\Psi^{0}(u)$ satisfies the following integral equation:

$$
\Psi^{0}(u)=H(\infty)-H(u)+\int_{0}^{u} \Psi^{0}(u-z) d H(z), \quad u \geq 0 .
$$

Proof. Equation (3.10) follows directly from (3.6).

Since $H(\infty)<1,(3.6)$ and $(3.10)$ are defective renewal equations.

Recalling that $H(\infty)=\rho+\lambda \mu / c$ and $\Phi^{0}(\infty)=1$, in the case of positive safety loading, we conclude that

$$
\begin{aligned}
& \Phi^{0}(0)=1-H(\infty)=(1-\rho)\left[1-\frac{\lambda \mu}{c(1-\rho)}\right], \\
& \Psi^{0}(0)=1-\Phi^{0}(0)=H(\infty) .
\end{aligned}
$$

Define $L_{\Phi^{0}}(s)$ to be the Laplace transform (LT) of $\Phi^{0}(u)$. Taking the LT of (3.6), we get

$$
L_{\Phi^{0}}(s)=\frac{\Phi^{0}(0)}{s\left[1-\mathrm{LS}_{H}(s)\right]}
$$

where $\mathrm{LS}_{H}(s)$ is the LST of $H(u)$. Using the standard properties of the transforms and their inversions leads to

$$
\Phi^{0}(u)=(1-H(\infty)) \sum_{n=0}^{\infty} H^{* n}(u), \quad u \geq 0,
$$


where $H^{* n}(u)$ means the $n$th convolution of $H(u)$ with itself. The same result can be derived by using the fact that renewal equation (3.6) has a unique solution; see for instance [9, Lemma 6.1.2].

Now we define

$$
H_{1}(u)=\frac{H(u)}{H(\infty)}=\frac{H(u)}{\rho+\lambda \mu / c},
$$

which is a proper probability distribution. For the nonruin probability, we have

$$
\Phi^{0}(u)=[1-H(\infty)] \sum_{n=0}^{\infty}[H(\infty)]^{n} H_{1}^{* n}(u), \quad u \geq 0,
$$

and for the ruin probability,

$$
\Psi^{0}(u)=[1-H(\infty)] \sum_{n=1}^{\infty}[H(\infty)]^{n} \bar{H}_{1}^{* n}(u), \quad u \geq 0,
$$

where $\bar{H}_{1}(u)=1-H_{1}(u)$.

In the above formula, we recognize a version of the Pollaczeck-Khinchin formula (or Beekman convolution formula); see [9].

According the definition of the relative safety loading (3.1), the following relations hold:

$$
1-H(\infty)=(1-\rho) \frac{\theta}{1+\theta}, \quad H(\infty)=\frac{1+\theta \rho}{1+\theta}
$$

So,

$$
\Psi^{0}(u)=(1-\rho) \frac{\theta}{1+\theta} \sum_{n=1}^{\infty}\left(\frac{1+\theta \rho}{1+\theta}\right)^{n} \bar{H}_{1}^{* n}(u), \quad u \geq 0 .
$$

In the case of $\rho=0, H_{1}(u)=H(u)=G(u)$ and (3.18) coincides with the ruin probability of the classical risk model.

Example 3.3. We consider the case of exponentially distributed claim sizes, that is, $F(u)=$ $1-e^{-u / \mu}, u \geq 0, \mu>0$. In this case, the integrated tail distribution $F_{I}(u)$ is exponential also and $H(u)=(\rho+\lambda \mu / c)\left[1-e^{-u / \mu}\right]$. So, $H_{1}(u)=1-e^{-u / \mu}$.

Taking into account that the $n$th convolution of $H_{1}(u)$ is an Erlang $(n, 1 / \mu)$ distribution with distribution function

$$
H_{1}^{* n}(u)=1-e^{-u / \mu} \sum_{j=0}^{n-1} \frac{(u / \mu)^{j}}{j !}
$$

relation (3.15) leads to

$$
\Phi^{0}(u)=1-H(\infty) \exp \left\{-\frac{1-H(\infty)}{\mu} u\right\} .
$$


The ruin probability in terms of the relative safety loading is given by

$$
\Psi^{0}(u)=\frac{1+\theta \rho}{1+\theta} \exp \left\{-\frac{1-\rho}{\mu} \frac{\theta}{1+\theta} u\right\} .
$$

If $\rho=0$, the result coincides with the example of Grandell [4, pages 5-6] related to the ruin probability for the classical risk model. The example is to be continued.

3.2. The stationary case. According to the arguments described by Grandell [4], if $\Phi^{0}(u)$ and $\Psi^{0}(u)$ are nonruin and ruin probabilities, respectively, in the ordinary case, then in the stationary case, we have the following result.

PROPOSITION 3.4. The nonruin probability $\Phi(u)$ and the ruin probability $\Psi(u)$ in the stationary case satisfy the integral representations

$$
\begin{aligned}
& \Phi(u)=\Phi(0)+\frac{\lambda}{c(1-\rho)} \int_{0}^{u} \Phi^{0}(u-z)(1-F(z)) d z \\
& \Psi(u)=\frac{\lambda}{c(1-\rho)}\left[\int_{u}^{\infty}(1-F(z)) d z+\int_{0}^{u} \Psi^{0}(u-z)(1-F(z)) d z\right] .
\end{aligned}
$$

Since $\Phi(\infty)=\Phi^{0}(\infty)=1$ when $c>\lambda \mu /(1-\rho)$, we have

$$
\Phi(0)=\frac{1-H(\infty)}{1-\rho}=1-\frac{\lambda \mu}{c(1-\rho)} .
$$

Taking the LP of (3.22) and applying (3.12), we have

$$
L_{\Phi}(s)=\frac{\Phi(0)}{s}+\frac{\lambda \mu}{c(1-\rho)} \mathrm{LS}_{F_{I}}(s) \frac{\Phi^{0}(0)}{s\left[1-\mathrm{LS}_{H}(s)\right]}
$$

Again, the standard properties of the transforms lead to

$$
\mathrm{LS}_{\Phi}(s)=\frac{1-H(\infty)}{1-\rho}+\frac{H(\infty)-\rho}{1-\rho} \operatorname{LS}_{F_{I}}(s)[1-H(\infty)] \sum_{n=0}^{\infty}\left[\operatorname{LS}_{H}(s)\right]^{n}
$$

So, the ruin probability in the stationary case is given by

$$
\Psi(u)=\frac{H(\infty)-\rho}{1-\rho}\left[\bar{F}_{I}(u)+F_{I}(u) *[1-H(\infty)] \sum_{n=1}^{\infty}[H(\infty)]^{n} \bar{H}_{1}^{* n}(u)\right],
$$

where $\bar{H}_{1}(u)=1-H_{1}(u)$ and $\bar{F}_{I}(u)=1-F_{I}(u)$.

In terms of the relative safety loading, the ruin probability is given by

$$
\Psi(u)=\frac{1}{1+\theta} \bar{F}_{I}(u)+\frac{1}{1+\theta} F_{I}(u) \frac{(1-\rho) \theta}{1+\theta} \sum_{n=1}^{\infty}\left(\frac{1+\theta \rho}{1+\theta}\right)^{n} \bar{H}_{1}^{* n}(u) .
$$


Example 3.5. Again, consider the case in which the claim amount distribution is exponential with mean value $\mu$. Applying the argument of Example 3.3 to the ruin probability (3.27) yields

$$
\Psi(u)=\frac{H(\infty)-\rho}{1-\rho} \exp \left\{-\frac{1-H(\infty)}{\mu} u\right\},
$$

and in terms of the relative safety loading,

$$
\Psi(u)=\frac{1}{1+\theta} \exp \left\{-\frac{1-\rho}{\mu} \frac{\theta}{1+\theta} u\right\} .
$$

In the case of $\rho=0,(3.30)$ coincides with that of Grandell [4, Example 6, page 69].

\section{The Cramér-Lundberg approximation}

4.1. The ordinary case. We return to the defective integral equation (3.10) for the ruin probability in the ordinary case. Assume that there exists a constant $R>0$ such that

$$
\int_{0}^{\infty} e^{R z} d H(z)=1
$$

where $H(z)$ is given by (3.4), and denote $h(R)=\int_{0}^{\infty} e^{R z} d F(z)-1$. Relation (4.1) is known as the Cramér condition. The constant $R$, if it exists, is called adjustment coefficient or Lundberg exponent. For any functions $f_{1}(x)$ and $f_{2}(x)$, we write $f_{1}(x) \sim f_{2}(x)$ for $x \rightarrow \infty$ if $\lim _{x \rightarrow \infty}\left(f_{1}(x) / f_{2}(x)\right)=1$.

Theorem 4.1. Let, for the Pólya-Aeppli risk model, the Cramér condition (4.1) holds and $h^{\prime}(R)<\infty$. Then

$$
\Psi^{0}(u) \sim \frac{\mu \theta A(\mu, \theta, R, \rho)}{A^{2}(\mu, \theta, R, \rho) h^{\prime}(R)-\mu(1+\theta)} e^{-R u},
$$

where $A(\mu, \theta, R, \rho)=(1-[1-\mu(1+\theta) R] \rho) /(1-\rho)$.

Proof. Multiplying (3.10) by $e^{R u}$ yields

$$
e^{R u} \Psi^{0}(u)=e^{R u}(H(\infty)-H(u))+\int_{0}^{u} e^{R(u-z)} \Psi^{0}(u-z) e^{R z} d H(z) .
$$

It follows from the definition of $R$ that integral equation (4.3) is a renewal equation.

The mean value of the probability distribution, given by

$$
G(t)=\int_{0}^{t} e^{R z} d H(z)
$$

is

$$
\int_{0}^{\infty} z e^{R z} d H(z)=\frac{\lambda}{c R}\left(1+\frac{c}{\lambda} R \rho\right) h^{\prime}(R)-\frac{1-\rho}{R(1+(c / \lambda) R \rho)} .
$$


Since

$$
\int_{0}^{\infty} e^{R u}(H(\infty)-H(u)) d z=\frac{1-H(\infty)}{R}=\frac{1-(\rho+\lambda \mu / c)}{R},
$$

by the key renewal theorem, we have

$$
\Psi^{0}(u) \sim \frac{(1+(c / \lambda) R \rho)((1-\rho)(c / \lambda)-\mu)}{(1+(c / \lambda) R \rho)^{2} h^{\prime}(R)-(1-\rho)(c / \lambda)} e^{-R u} .
$$

Taking into account that $c / \lambda=\mu(1+\theta) /(1-\rho)$, we get the Cramér-Lundberg approximation in terms of the relative safety loading given by (4.2).

If $\rho=0, A(\mu, \theta, R, 0)=1$ and (4.2) coincides with the Cramér-Lundberg approximation for the classical risk model [4, page 7].

Example 4.2. If we take $F(x)=1-\exp (-x / \mu)$, then $h(R)=\mu R /(1-\mu R)$. The constant $R$ is a positive solution of the equation

$$
\left(1+\frac{c}{\lambda} R \rho\right) \frac{\mu R}{1-\mu R}=(1-\rho) \frac{c}{\lambda} R,
$$

that is,

$$
R=\frac{1-\rho}{\mu}\left(1-\frac{\lambda \mu}{c(1-\rho)}\right)=\frac{1-\rho}{\mu} \frac{\theta}{1+\theta},
$$

and $A(\mu, \theta, R, \rho)=1+\theta \rho$.

So, the Cramér-Lundberg approximation is exact when the claims are exponentially distributed and given by

$$
\Psi^{0}(u) \sim \frac{1+\theta \rho}{1+\theta} e^{-R u} .
$$

4.2. The stationary case. We write integral representation (3.23) for the ruin probability in the stationary case in the following equivalent form:

$$
\Psi(u)=\frac{\lambda \mu}{c(1-\rho)}\left[\bar{F}_{I}(u)-\int_{0}^{u} \Psi^{0}(u-z) d \bar{F}_{I}(z)\right] .
$$

Taking into account the Cramér-Lundberg approximation in the ordinary case, we have

$$
\Psi(u) \sim \frac{(1-\rho)(c / \lambda)-\mu}{(1+(c / \lambda) R \rho)^{2} h^{\prime}(R)-(1-\rho)(c / \lambda)} e^{-R u},
$$

and in terms of the relative safety loading,

$$
\Psi(u) \sim \frac{\mu \theta}{A^{2}(\mu, \theta, R, \rho) h^{\prime}(R)-\mu(1+\theta)} e^{-R u} .
$$


In the case of $\rho=0$, asymptotic relation (4.13) coincides with the Cramér-Lundberg approximation in the classical risk model.

Example 4.3. Again, in the case of exponentially distributed claim sizes, the CramérLundberg approximation is exact and is given by

$$
\Psi(u) \sim \frac{1}{1+\theta} e^{-R u},
$$

where $R$ is a positive solution of (4.8).

\section{Comparison of ruins}

The value of the Lundberg exponent is a measure of the dangerousness of the risk business. Relative to this measure, the ordinary and the stationary cases are equally dangerous; see $[4$, page 70$]$.

We will compare the Pólya-Aeppli risk model with the corresponding classical model. According to the definition given by De Vylder and Goovaerts [2], corresponding risk models are models with the same claim size distribution, the same expected number of claims in any time interval $[0, t]$, the same security loading, and the same initial risk reserve. The classical risk model corresponding to the Pólya-Aeppli risk model has a Poisson counting process with intensity $\lambda /(1-\rho)$ and a relative safety loading given by (3.1). The interarrival times are exponentially distributed with parameter $\lambda /(1-\rho)$. Now we need the following lemma [4].

LEMMA 5.1. Let $T^{1}$ and $T^{2}$ be two r.v.'s representing the interarrival times of two risk models. Let $R_{1}$ and $R_{2}$ be the corresponding Lundberg exponents. If $\operatorname{LS}_{T^{1}}(s) \leq \operatorname{LS}_{T^{2}}(s)$ for all $s>0$, then $R_{1} \geq R_{2}$.

Let $T^{\mathrm{cl}}$ and $T$ be two r.v.'s representing the interarrival times of the corresponding classical risk model and the Pólya-Aeppli risk model. Then

$$
\mathrm{LS}_{T^{\mathrm{cl}}}(s)=\frac{\lambda /(1-\rho)}{s+\lambda /(1-\rho)}, \quad \mathrm{LS}_{T}(s)=\rho+(1-\rho) \frac{\lambda}{s+\lambda}
$$

It is easy to see that $\mathrm{LS}_{T^{\mathrm{cl}}}(s) \leq \mathrm{LS}_{T}(s), s>0$. Applying Lemma 5.1, it follows that $R^{\mathrm{cl}} \geq R$, where $R^{\mathrm{cl}}$ is the Lundberg exponent for the classical model. This means that the PólyaAeppli risk model is more dangerous than the corresponding classical model.

The comparison of the exact ruin probabilities depends on the claim size distribution. We can compare analytically the particular cases of exponentially distributed claim sizes. At first, we compare the ruin probability of the stationary case (3.30) with the ruin probability of the corresponding classical risk model given by

$$
\Psi_{\mathrm{cl}}(u)=\frac{1}{1+\theta} \exp \left\{-\frac{1}{\mu} \frac{\theta}{1+\theta} u\right\} .
$$

It is easy to see that for all $u \geq 0$,

$$
\Psi(u) \geq \Psi_{\mathrm{cl}}(u)
$$


On the other hand, the comparison between (3.21) and (3.30) states that for all $u \geq 0$,

$$
\Psi^{0}(u) \geq \Psi(u)
$$

So, in the case of exponentially distributed claim sizes, the most dangerous is the ordinary case and the less dangerous is the classical risk model.

Comparing the ruin probabilities, it is natural to mention the difference between the Pólya-Aeppli risk model and the classical model in the case of $\rho=0$. It suffices to compare the ruin probability in the stationary case (3.30) and the corresponding ruin probability,

$$
\Psi_{\{\rho=0\}}(u)=\frac{1}{1+\theta_{\{\rho=0\}}} \exp \left\{-\frac{1}{\mu} \frac{\theta_{\{\rho=0\}}}{1+\theta_{\{\rho=0\}}} u\right\},
$$

in the case of $\rho=0$. From (3.1), it follows that

$$
\theta_{\{\rho=0\}} \geq \theta
$$

Then for all $u \geq 0$, we have

$$
\Psi_{\{\rho=0\}}(u) \leq \Psi(u) .
$$

In this case, again the Pólya-Aeppli risk model is more dangerous than the classical risk model.

It is useful to analyze the differences between the ruin probabilities even in the particular cases. The distributions of the interarrival times of the corresponding models have the same expected values. In the Pólya-Aeppli risk model, we have $P\left(T_{2}=0\right)=\rho>0$, that is, the probability that the claims arrive simultaneously is not equal to zero. This can cause the ruin and the ruin probability is greater.

\section{Acknowledgments}

The author would like to thank Hanspeter Schmidli for useful comments related to this paper. Furthermore, he would like to thank the referee for remarks leading to an improvement of the presentation of the paper. This work is supported by FAPESP Grant 98/05810-9, Brazil.

\section{References}

[1] S. Asmussen, Ruin Probabilities, Advanced Series on Statistical Science \& Applied Probability, vol. 2, World Scientific Publishing, New Jersey, 2000.

[2] D. C. M. Dickson, On a class of renewal risk processes, N. Am. Actuar. J. 2 (1998), no. 3, 60-73, with discussion by F. E. De Vylder and M. J. Goovaerts and a reply by the author.

[3] W. Feller, An Introduction to Probability Theory and Its Applications. Vol. II, 2nd ed., John Wiley \& Sons, New York, 1971.

[4] J. Grandell, Aspects of Risk Theory, Springer Series in Statistics: Probability and Its Applications, Springer-Verlag, New York, 1991.

[5] N. L. Johnson, S. Kotz, and A. W. Kemp, Univariate Discrete Distributions, 2nd ed., Wiley Series in Probability and Mathematical Statistics: Applied Probability and Statistics, John Wiley \& Sons, New York, 1992. 
[6] V. Kalashnikov and R. Norberg, Mathematical Methods in the Ruin Probability Theory, Lecture Notes, Copenhagen University, Copenhagen, 1998.

[7] L. Minkova, A family of compound discrete distributions, C. R. Acad. Bulgare Sci. 54 (2001), no. 2, 9-12.

[8] A generalization of the classical discrete distributions, Comm. Statist. Theory Methods 31 (2002), no. 6, 871-888.

[9] T. Rolski, H. Schmidli, V. Schmidt, and J. Teugels, Stochastic Processes for Insurance and Finance, Wiley Series in Probability and Statistics, John Wiley \& Sons, Chichester, 1999.

Leda D. Minkova: Faculty of Mathematics and Informatics, Sofia University "St. Kliment Ohridski," 5 James Bourchier Boulevard, 1164 Sofia, Bulgaria

E-mail address: leda@fmi.uni-sofia.bg 


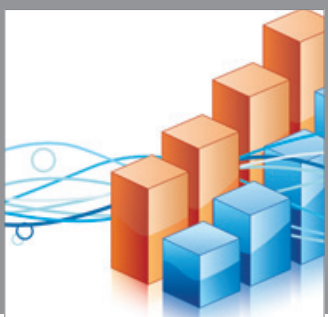

Advances in

Operations Research

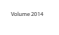

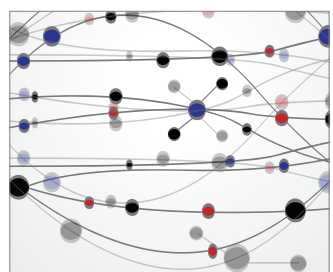

\section{The Scientific} World Journal
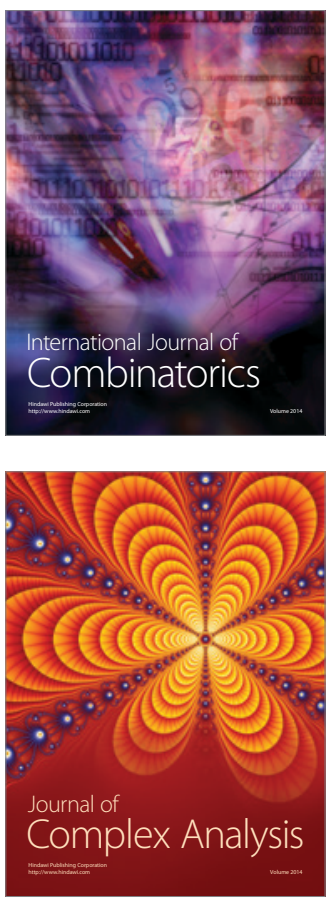

International Journal of

Mathematics and

Mathematical

Sciences
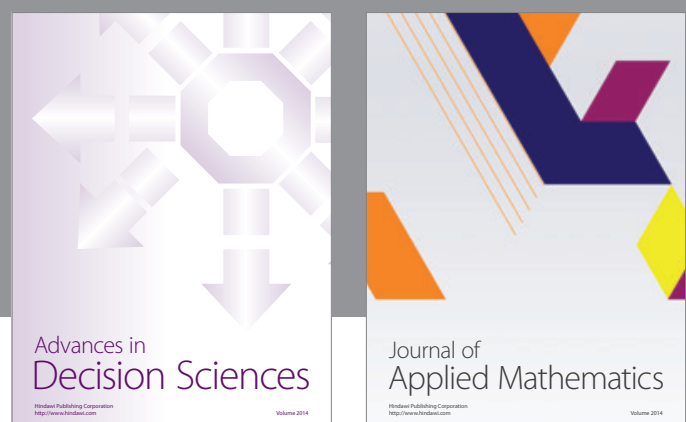

Journal of

Applied Mathematics
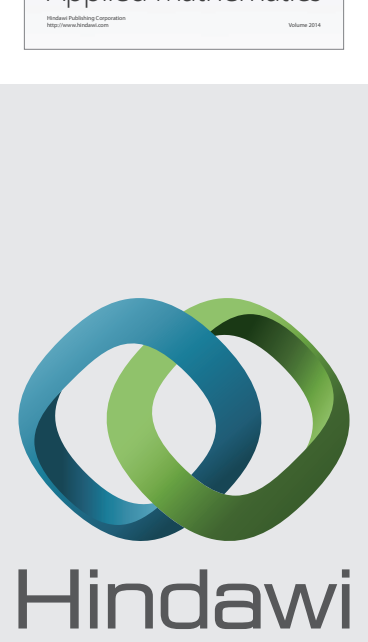

Submit your manuscripts at http://www.hindawi.com
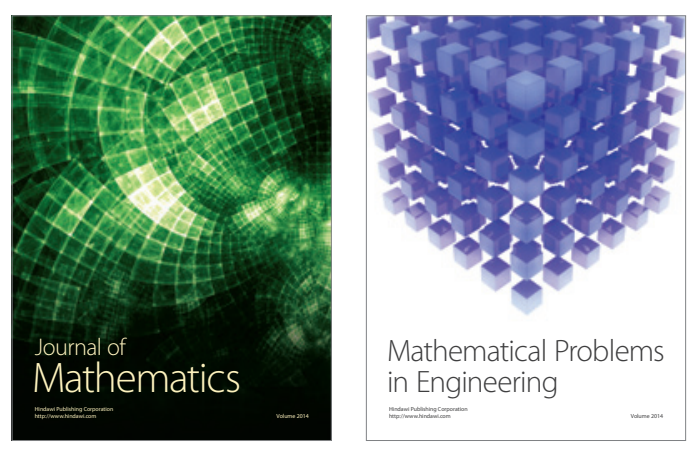

Mathematical Problems in Engineering
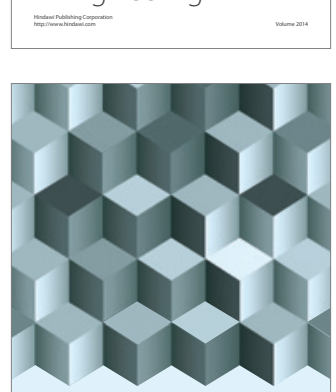

Journal of

Function Spaces
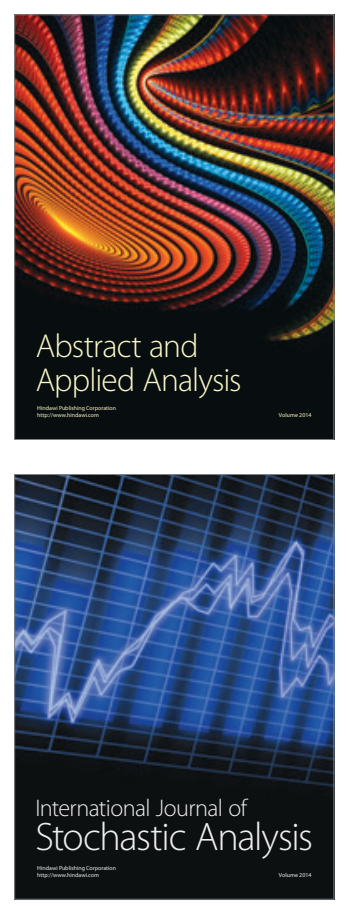

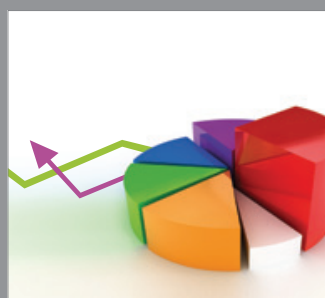

ournal of

Probability and Statistics

Promensencen
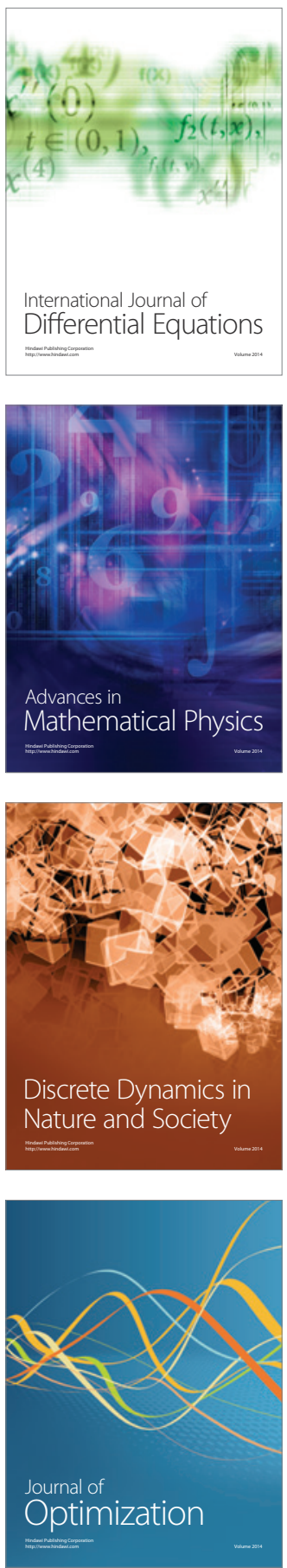